\title{
Elements of a Theory of Nonphysical Agents in the Physical World
}

\author{
Uwe Meixner*
}

Received: 17 May 2018 / Accepted: 3 December 2018

\begin{abstract}
This paper shows that there is a quantum-physical and evolution-biological perspective for (libertarian) free will, and that the so-called scientific arguments against it are in reality metaphysical arguments and insufficient. The paper also develops the idea of a nonphysical organ of higher organisms: the Domindar (Detector of macroscopic indetermination, and restrictor).

Keywords: Brain; chance; choice; consciousness; determinism; Domindar; indeterminism; nonphysical cause; quantum physics; principle of causal closure; principle of sufficient cause; subject of consciousness and of physical action.
\end{abstract}

\section{The present situation, and a plan of action}

In recent years things have not looked good for human 'free will.' Based on neurophysiological facts, its existence has been denied, or in other words: it has been denied that we ever are in the full and proper sense originators of our own physical actions. In this paper, I propose to show that the scientific (quantum-physical and evolution-biological) facts do not offer a sufficient

* University of Augsburg

Department of Philosophy, University of Augsburg, Universitätsstr. 10, D-86159 Augsburg, Germany

$\bowtie$ uwe.meixner@phil.uni-augsburg.de

(C) The Author. Journal compilation (c) The Editorial Board, Organon F.

This article is distributed under the terms of the Creative Commons Attribution-NonCommercial 4.0 International Public License (CC BY-NC 4.0). 
reason for a denial of 'free will.' On the contrary, those facts strongly suggest that we (and the subjects of other higher animals) do at least sometimes act, in the full and proper sense, in the realm of the physical, that is: they strongly suggest that we make genuine (hence also truly decisive) decisions between alternatives of being, between ways for the physical world to be. We have something to decide, and we exist for deciding something.

\section{An important distinction: indeterminism and resolution of indeterminism}

I begin with a fundamentally important distinction. Physical indeterminism and the resolution of physical indeterminism are two different things. Physical indeterminism is given at a time $t$ if, and only if, beginning with time $t$ there are - the completed physical past up to $t$ notwithstanding - several physically possible further courses of the physical world (at least two, if not more). That physical indeterminism occurs at some times, perhaps at all times, is today a widely accepted lesson from quantum physics. This lesson, however, is not a necessary or logical consequence of quantum physics. It certainly is a matter of its interpretation - of an interpretation of quantum physics, however, which is natural and plausible and which has almost completely prevailed against Einsteinian dreams of a restoration of physical determinism, and against the Bohmian alternative interpretation which safeguards the old determinism. In what follows, I presuppose the existence of physical indeterminism.

Now, resolution of physical indeterminism always occurs when physical indeterminism occurs; for always the physical world continues in a determinate way: in precisely one of the ways physically possible in the indeterministic situation. ${ }^{1}$ Always the physical indeterminacy is replaced by determinacy. There are two possible manners of a resolution of physical

\footnotetext{
1 Some philosophers favor the so-called many-worlds interpretation of quantum physics [see (DeWitt and Graham 1973)], according to which all the ways which are physically possible in a given indeterministic situation are actualized. I do not believe that this view has enough metaphysical, let alone empirical, warrant to be credible. It brings along with it altogether too many unverifiable actualities, and mainly, it
} 
indeterminism, of the replacement of physical indeterminacy by determinacy: one, by ontic chance, hence without any sufficient cause; two, by a sufficient nonphysical cause. Let us take note: The existence of physical indeterminism does not logically entail the existence of ontic chance in the physical world; for physical indeterminism and the resolution of physical indeterminism are, on the one hand, two different things, and on the other hand, the resolution of physical indeterminism need not always, or even sometimes, come about by ontic chance.

\section{Two different metaphysical principles - two different outcomes}

It is not a logical consequence of quantum physics that physical indeterminism occurs; it is, even more emphatically, not a logical consequence of quantum physics that the resolution of physical indeterminism always occurs via ontic chance. This latter consequence follows only if one adds a principle to quantum physics which is not a principle of physics but a principle of a specific kind of metaphysics: the principle of the causal closure of the physical world, which principle says that every physical event that has a sufficient cause at all also has a sufficient physical cause. ${ }^{2}$ Assume there is

seems, in order to get rid of the wonder that is provoked when one sticks to the (truly) empirical facts, the facts of appearance: As far as we know from appearances, just one of the physically possible ways is actualized out of every indeterministic situation.

2 This is a (logically) weak version of the closure principle; it follows logically from (logically) stronger versions of that principle. A stronger version which is so strong as to be directly refuted by the admittance of ontic chance into the physical world is this: Every physical event has a sufficient physical cause. Another stronger version, however, is not refuted in this manner: Every sufficient cause of a physical event is itself physical. In (Kim 1993, 280) the following version of the "causal closure of the physical domain" can be found: Any physical event that has a cause at time $t$ has a physical cause at $t$, which can be interpreted in such a manner as to be roughly equivalent with the above-presented weak version. Its many versions notwithstanding, in (Popper and Eccles 1977, 51) what needs to be said about the principle of causal closure in the first place has already been said: "[T] he physicalist principle of the closedness of the physical [world] [...] is of decisive importance, and I take it as the 
physical indeterminism at $t$; hence beginning with time $t$ there are several physically possible further courses of the physical world, the entirety of the physical past up to $t$ notwithstanding. Precisely one of these further courses becomes actual. How does this come about? The answer is, prima facie, unclear. What is clear, however, is this: in whichever manner the resolution of the physical indeterminism at hands comes about, there is, in any case, a physical event beginning at time $t$ without sufficient physical cause- since even the entire physical past up to $t$ is not a sufficient physical cause of it. On the basis of the principle of the causal closure of the physical world, it follows for such an event that it has no sufficient cause at all, hence that it happens by ontic chance.

Unfortunately — or fortunately (depending on one's metaphysical point of view) - this conclusion is by no means indisputable. For if, instead of the principle of the causal closure of the physical world, one assumes the principle of sufficient cause-Every event has a sufficient cause ${ }^{3}$ - as a metaphysical addition to quantum physics, then it follows that the event in question - the event beginning at time $t$ without sufficient physical causedoes have a sufficient cause, but, of course, a nonphysical one.

\section{A brief assessment of conflicting principles}

The principle of the causal closure of the physical world has no greater right to be believed in than the principle of sufficient cause, neither systematically nor historically. On the contrary, the principle of sufficient

characteristic principle of physicalism or materialism." This principle belongs to metaphysics, not to physics.

3 The principle of sufficient cause is not the - better-known-principle of sufficient reason: For every existing entity there is a sufficient reason of its existence. The principle of sufficient cause is, however, a logical consequence of the principle of sufficient reason; this is so in virtue of the fact that every event is an existing entity, and that if there is a sufficient reason for the existence of $x$, that then there also is a sufficient cause for the existence of $x$ (for this to be true, it need not be true that every reason is a cause). A near relative of the principle of sufficient cause is relied on in William Craig's modernizations of the cosmological argument; see, for example, (Craig and Sinclair 2012). 
cause is metaphysically more neutral, hence in a certain sense more rational than the closure principle (not long ago, the principle of sufficient cause still enjoyed the dignity of a quasi-logical principle), and historically it is, of course, by far the more respected postulate. Mainly one reason seems to speak for the principle of the causal closure of the physical world, and it is not a rational reason: those who believe in it have an intellectual fear of nonphysical causes of physical events, they even fear a nonphysical influence on the physical. Even theologians, nowadays, seem to fear a causality of the nonphysical that touches the physical world; such is the modern mentality. One cannot well say - although many do say it - that there is no empirical evidence for nonphysical causes of physical events; for if one assumes the principle of sufficient cause instead of the principle of causal closure, then the interpretation of the physical facts - of the quantum-physical facts - will be quite different than it was before: where previously one saw physical events without any sufficient cause, one now sees physical events with a sufficient nonphysical cause. Of course, strictly speaking, one does not 'see' either the one or the other; strictly speaking, one judges the empirical situation differently by adhering to different metaphysical interpretations of it.

The principle of sufficient cause requires that the resolution of physical indeterminism always occur by a nonphysical sufficient cause; the principle of the causal closure of the physical world requires that this resolution always be by ontic chance, without any sufficient cause. In what follows, I proceed on the assumption that some, even many, resolutions of physical indeterminism occur by nonphysical causes; in doing so, I position myself against the principle of causal closure, consider it false - without, however, wishing to wholly exclude ontic chance as a means of the resolution of physical indeterminism. The principle of sufficient cause, too, is, perhaps, false, and what follows below does not rely on the truth of the principle of sufficient cause. $^{4}$

4 What follows below is, moreover, essentially different from the proposal of the physicist Eugene Wigner. Wigner did assume an influence of consciousness on the physical; he, however, did not assume an influence of the will on the physical. On Wigner's proposal, see (Barrett 2014, 67-70); according to Wigner, "conscious apprehension causes collapses [of the wave function; of the quantum-physical 


\section{Indeterminism and the agency of natural living beings}

What is the import of all this for the agency of natural living beings? The agency of natural living beings is their agency in the physical macroworld. The presupposition for the existence of such agency is not only the existence of physical indeterminism simpliciter, but also the existence of physical indeterminism in the physical macro-world, to boot, of such indeterminism as is detectable by natural living beings. Only if there are situations with alternative possibilities - indeterministic situations - in the physical macro-world and only if they can be detected by natural living beingsonly then can such beings contribute to the resolution of such situations (for example, in order to obtain a biological advantage). In this, precisely, does the agency of natural living beings consist: in contributing to the resolution, the ontic deciding, of macrophysical indeterministic situationsnormally, with the aim of obtaining a biological advantage.

What are the indications that there are indeterministic situations in the physical macro-world which can be detected by natural living beings? What are, in other words, the indications that for some times $t$ the following is true: there are several physically possible further courses of the physical macro-world beginning with $t$, and in such a manner that this situation can be found out by a natural living being?

One indication is that the physical macro-world strikes us - and perhaps not only us - as being to a considerable extent contingent. In every reflective second of our waking life we have, looking back at the past of the physical macro-world, the consciousness that things could have been otherwise than they actually were, that we, in particular, could have acted differently than we did; looking ahead at the future of the physical macro-world, we have the consciousness of being able to act like this, or otherwise; more generally speaking, we have the consciousness that things can be like this in the future, or otherwise. Those who assume determinism for the physical macroworld must consider the apparent contingency of the physical macro-world as one gigantic, incessant illusion - an illusion which cannot be eliminated; which can only be uncovered. But how plausible, really, is this stance? Why

state]" (Barrett 2014, 70). As will become clear below, this is not the view here proposed. 
the consciousness of contingency, why even consciousness at all, if the physical macro-world is ruled by determinism? If it is ruled by determinism, consciousness is pure luxury, extravagancy. Sometimes, indeed, it does happen that biological evolution treats itself to a luxury, but not to a luxury which has such a high price: the large amount of energy that the central nervous system consumes in the production of consciousness, including the insistent, imperturbable consciousness of contingency.

Another indication of the existence of indeterministic situations in the physical macro-world is this: there seem to exist in the physical macroworld goings-on that can be correctly described as 'competition,' 'struggle,' 'fight.' Who believes in determinism in the physical macro-world must, however, deny the existence of such goings-on. True fights cannot exist for a determinist. Two soccer teams, it is said, fight for victory. Not so; if determinism rules in the physical macro-world, then who will win is determined even before the two teams begin to play - and, rightly considered, the verb 'to win' must here be put in scare quotes. Two males, it is said, fight over a female. Not so; if determinism rules in the physical macroworld, then what happens in this latter case is, as in the case of the soccer game, only the masquerade of a fight, only the masquerade of a competition. Everything is already determined, everything has already been decided beforehand, nothing is open anymore -if, if indeed, it is really the case that determinism rules in the physical macro-world. There are only 'rigged games' then, and also the very serious game of life, the so-called 'struggle for existence,' is a 'rigged game' then, not a true struggle; it is, then, a bad, not to say evil, joke, considering that, for the antagonists in that game, it feels exactly as if it weren't 'rigged,' exactly as if it were an open struggle and they were fighting, really fighting for a good outcome for themselves.

\section{Micro- and macro-indeterminism, and the brain}

It is not only morality which is rendered absurd by determinism in the physical macro-world, it is biology, too (and, by the way, philosophy as well; for what is the point in discussing anything at all if all our voiced opinions, whichever they may be, are already determined to be voiced by us even before we began to exist?). And this is nothing less than a reductio 
ad absurdum of physical macro-determinism-albeit, as we all know, not a generally accepted reductio. Indeterminism in the physical micro-world, and hence also, as a logical consequence, physical indeterminism simpliciter, do indeed enjoy widespread credence - due, firstly, to the almost general acceptance of quantum physics and, secondly, to the prevalence of its standard interpretation. But indeterminism in the physical macro-world is still widely rejected. With respect to the physical macro-world, determinism of the Newtonian-Laplacian stamp is still widely accepted; the explanation usually given is this: microphysical indeterminism cannot play any macrophysical role, cannot magnify itself into the macrophysical realm because of the massive and unavoidable disturbances produced by the natural environment.

It is, therefore, for many people as if there were indeed two parallel physical worlds: one microphysical, in which indeterministic situations occur, as can be found out by complicated procedures; and one macrophysical, in which no indeterministic situations occur; in which, in any case, noneit is said - are scientifically detectable. In fact, this latter position is nowadays almost invariably assumed by one of the metaphysically interested sides: by those who have a materialist-naturalistic metaphysical outlook. Invariably they hold that indeterministic situations in the physical macroworld are scientifically undetectable, and invariably this is taken as evidence for there being no such situations. However, assuming for the sake of the argument that there are such situations, how could they be scientifically detected? They would have to be detected after they are already over, after they have already been resolved into determinacy: by proving - that is, by ascertaining beyond scientific doubt - that certain physical macro-events have no sufficient physical cause. Now, one encounters physical macroevents in huge numbers which, in fact, do not seem to have a sufficient physical cause chaotic goings-on of the most various kinds, so-called chance processes, among them the familiar die-throws and coin-throws, with their concluding events that serve, for the purposes of everyday life, as chance events. Yet, the step from 'seems not to have a sufficient physical cause' to 'does not have a sufficient physical cause' is never ever, under no circumstances, taken by the metaphysically prejudiced-because they suppose a priori that there must be a sufficient physical cause even though 
none is apparent. Thus, endeavoring to prove scientifically that there are physical macro-events without sufficient physical cause - in order to prove that there are indeterministic situations in the physical macro-world-encounters in the minds of many people from the start an insurmountable obstacle: a priori, and quite unscientifically, such events and such situations are not accorded a fair chance of existing.

They should be given such a chance. Is it not to be expected that a large microphysical indeterminism - one that involves large numbers of elementary particles - will issue into a macrophysical indeterminism? Is it not to be expected that on intricate and involved paths even a small microphysical indeterminism will lead to a macrophysical one? We are, after all, not dealing with two parallel physical worlds, we are dealing only with one physical world; the distinction between microphysical and macrophysical has no separating significance. This one physical world is ruled by the laws of quantum physics, not by those of nineteenth-century physics. Brain scientists like to emphasize that brain processes obey without exception the laws of physics. This is, of course, true; for brain processes are physical processes. But the laws they obey are the laws of quantum physics, not the laws of the old, deterministic physics.

Therefore, also in the brain we must expect to encounter physically spontaneous physical events: physical events without a sufficient physical cause; and that some such events-as brain processes (not already as processes in the brain: most processes in the brain are microphysical) — would be physical macro-events, would still be physical macro-events. Physically spontaneous physical macro-events in the brain would, however, be the unmistakable indicators of the occurrence of brain-centered macrophysical indeterministic situations.

\section{The inconclusiveness of the Libet-experiment}

Some time ago, the result of the so-called Libet-experiment produced considerable reverberations. The readiness potential for a physical action, which is given in the brain already some time before one is conscious of deciding 'to do the deed,' was regarded by some as a refutation of free will. 'Look here,' they rejoiced (the verb 'to rejoice' is not as inappropriate as it 
may seem at first sight), 'before you believe that you are deciding, the brain has already decided. You didn't decide anything, or in any case you did not genuinely decide anything, for your decision, coming too late, was, in any case, not the truly decisive one.' What seems to have been deigned attention only seldom, if at all, was the question of what produces the readiness potential in the first place. So eager was one to consider the brain a deterministic automaton that hardly anybody - perhaps nobody, I don't know - considered the possibility that the brain processes which contribute to the readiness potential are physically spontaneous processes-that is, physical events without a sufficient physical cause. One has not excluded this possibility, and I, for one, wouldn't know how it could be excluded. If the brain processes in question are in fact physical events without a sufficient physical cause, then there are, concerning their being caused or not (their being made to come about or not), only two basic options: either they do not have any sufficient cause, or they do have a nonphysical sufficient cause. In both cases, the occurrence of a brain-centered macrophysical indeterministic situation is indicated. As long as physics remains pure natural science, physics excludes neither one of the two mentioned options. Physics, as long as it stays free of metaphysics, gives both ontic chance and nonphysical causality a chance: in general, and in particular with respect to brain processes. Nonphysical causality, however, is what must interest us when considering agency; for agency is not a matter of chance.

\section{Subjects of consciousness and of physical action}

Let us consider a hypothesis, or rather, a network of hypotheses.-The waking subject of consciousness of a natural consciousness-endowed living being is, at the same time, the subject of physical action of that living being. Consciousness - among other things it offers in the line of service - points out to the waking subject, directly in perception (against the background of past experience), and by and large correctly, macrophysical indeterministic situations, especially such on the resolution of which the subject can exercise some influence. Moreover, consciousness proposes to the waking subject 'guidelines' (usually called 'motives') for the exercise of its influence, in case 
that influence is actually exercised: appetitive or aversive emotions and sensations, (conscious) needs and drives. But these 'guidelines,' even if they are considered in their entirety, are at least sometimes non-determinative: the subject remains - at least sometimes - up to a certain degree the sovereign of its physical actions. If it were not so, subjects of consciousness and of physical action would be biological superfluities. However, a subject of consciousness and of physical action that belongs to a natural living being (in what follows, I exclusively consider subjects of consciousness and of physical action that belong to a natural living being) is so far from being a biological superfluity that it is a nonphysical organ of its organism: an organ which serves, like every organ of the organism, the organism's life, especially its preservation. Consider what happens if this nonphysical organ goes temporarily out of service, or does not fulfill its tasks satisfactorily: the organism is rendered less protected, or is left entirely unprotected.

The service, then, that a subject of consciousness and of physical action renders to the life of its organism consists in this: to the advantage of its organism, it contributes to the resolution of some of the macrophysical indeterministic situations it detects and on the resolution of which it can exercise an influence. The agency of a subject of consciousness and of physical action is, precisely, the restriction of macrophysical indeterminism. Consider that every macrophysical indeterministic situation consists in a set of several possibilities: the physically possible further courses of the macrophysical world, beginning with a time $t$. From some such sets a subject of consciousness and of physical action selects a non-empty proper subset. And if a subject of consciousness and of physical action resolves a macrophysical indeterministic situation already all by itself, then it is precisely a singleton set which that subject selects from the pool of possibilities. In any case, the subject of consciousness and of physical action exercises a causal influence, a greater or smaller one. Its choice has an ontic effect, and it is not a blind choice: the subject of consciousness and of physical action chooses in the light of its consciousness, in the light of its outer and inner perceptions, in the light of its 'guidelines of the will,' in the light of its rational considerations (if there are such). Often its choice is, nevertheless, to a certain degree arbitrary, sometimes entirely arbitrary. But arbitrariness does not turn choice into ontic chance. Ontic chance and choice exclude each other-even 
if the choosing is arbitrary. If what I do is my choice, I am choosing what I do; if what I do is an ontic chance, nobody is choosing what I do. And if my choice is completely non-arbitrary (because consciousness clearly and distinctly indicates what is best for the best of reasons, and because I, a rational subject of consciousness and of physical action, follow this indication unhesitatingly and unwaveringly), then the perfect rationality of my choice does not turn my choice into a case of passive determination. Passive determination, too, and choice exclude each other. If what I do is my choice, I am choosing what I do; if what I do is due to passive determination, I am not choosing what I do; for under passive determination - not determining, but being determined-I do not have a choice.

The agency of the subject of consciousness and of physical action for the organism does not take place beside or behind the organism; it is not as if such a subject were a sort of guardian angel for the organism. Rather, the subject of consciousness and of physical action emerges together with its consciousness and its powers from the nervous system of the organism, mainly from the brain, and it is in its existence and in many details of its existence nomologically bound - bound by the laws of nature - to neuronal functions. It is, however, not determined by the nervous system in every respect; for that would mean that it is an epiphenomenon of the neuronal and hence a biological superfluity; but nature is not fond of superfluities. Rather, the subject of consciousness and of physical action is a highly useful-biologically useful, hence evolution-favored - detector of macroscopic indetermination, and restrictor, in short: a Domindar. ${ }^{5}$

5 The idea of Domindars is developed and justified in (Meixner 2006) and (Meixner 2008). That Domindars - subjects of consciousness and of physical action - emerge from organisms, in particular, from their nervous systems, is far from providing an ultimate metaphysical explanation of them. The main issue in their ultimate metaphysical explanation would be whether the emergence of Domindars is natural and without a supernatural grounding: is effected on the basis of uncreated natural laws and circumstances; or is natural and with a supernatural grounding: is effected on the basis of God-created natural laws and circumstances. My sympathies are with the latter, but one certainly needn't be an orthodox theist to believe in the emergence of Domindars. A wholesale rejection of psycho-physical emergentism"The physical cannot beget the nonphysical! In any case, it is incomprehensible 
The true relationship between the organism's subject of consciousness and of physical action - the Domindar - and the organism's nervous system can be illustrated cum grano salis by an analogy. A pianist is not able to play the piano without a piano, and if she plays the piano, then much in her playing is determined by the piano she plays on. But not everything in the pianist's piano-playing is determined by the piano played on: not which pieces of music the pianist plays, and not which interpretation she accords to them; also not how well the pianist plays - insofar as this is up to her. The piano is a necessary instrument of the pianist qua pianist; the nervous system - with the brain at its center - is a necessary instrument of the Domindar qua Domindar. Without their respective instruments they cannot - for necessary, compelling reasons - do what is their calling. However, this leaves entirely undiminished the fact that the pianist cannot be reduced to a piano, and that the Domindar is irreducible to a nervous system.

At one point, especially, this analogy is not perfect: a pianist is not a product - let alone a nonphysical product - of the instrument she plays on, whereas a Domindar, regarding its existence and the range of its powers, is indeed a nonphysical product of the very thing that the Domindar "plays on' (so to speak), after having been 'installed.' The relationship between Domindar and nervous system is much closer than the relationship between pianist and piano - so close that the nervous system, especially the brain, can well be called an instrumental Domindar.

How does this instrument function? At bottom, it functions not fundamentally unlike the way a piano functions. The pianist reads the musical score, and on the basis of this cognizance, she presses selectively the piano keys as she wishes and thinks fit; the internal mechanism of the piano translates the resulting patterns of key-pressings into the sequences of sounds intended by the pianist. Analogously: The subject of consciousness and of physical action (the Domindar) reads in its consciousness, and on the basis of this cognizance it contributes by informed choice to the resolution of an

how it could" - is not recommendable: The facts of mind-brain-interaction undeniably show that consciousness - something non-abstract and nonphysical - nomologically depends in many important ways on the physical. Why should it not also come from it (whether with the help of God or without), without being determined by it?

Organon F 26 (1) 2019: 104-121 
extra-neuronal macrophysical indeterministic situation detected by it (for example, 'Left? Right? Or straight on?'); the internal mechanism of the nervous system translates the resulting physically spontaneous (but Domindar-determined) brain process - the immediate effect of the subject's choosing - into the subject-intended (as such, extra-neuronal) restriction of precisely the macrophysical indeterministic situation in question.

\section{Domindar vis-à-vis extra-neuronal macrophysical indeterministic situation}

An extra-neuronal macrophysical indeterministic situation has - in its relation to a cerebrated natural living being "which can do something about it' - a cerebral, a neuronal, and an extra-neuronal aspect. Corresponding to this, the contribution of the living being's subject of consciousness and of physical action to the resolution of that situation has, likewise, a cerebral, a neuronal, and an extra-neuronal aspect. The cerebral aspect - the physically causeless brain event caused by the nonphysical subject of consciousness and of physical action - is that subject's originative (and properly own) causal influence; the rest is a (sometimes misfiring) causal projection into the larger macrophysical environment by means of 'automatic' neuronal and muscular electro-chemistry and mechanics. However, what is important to the subject of consciousness and of physical action is precisely this projection, and mainly the extra-neuronal part of it (because that part is what is intended by the subject): the bodily movement (whether in flight, fight, or other life-relevant situation).

The mechanisms of the event-causal connection between the cerebral and the extra-neuronal aspect-via the neuronal aspect - are nowadays well understood. I need not emphasize how precarious, how endangered this connection is in every cerebrated natural living being, and in particular in every human being. I also need not emphasize that it seems mysterious how the nonphysical subject of consciousness and of physical action - the Domindar - manages to decide a macrophysical indeterministic situation at least to such an extent partially that a physically spontaneous brain event comes about, an event which is not an event of ontic chance 
(i.e., without any sufficient cause at all) but caused by the Domindar, and truly decisively caused by the Domindar: without superfluity of the Domindar's causality. ${ }^{6}$ How this is effected seems mysterious to everybody. ${ }^{7}$ It should be noted, however, that there is little rationality in seeking to turn the mysterious how of the originative, seminal agency of the subject of consciousness and of physical action into, so to speak, a rope wherewith to strangle that subject philosophically. Generally speaking, the mysteriousness of the state of affairs that X makes $\mathrm{E}$ happen-with regard to how $\mathrm{X}$ makes $\mathrm{E}$ happen-neither entails the non-obtaining of that state of affairs, nor the nonexistence of X. There is, moreover, simply no doubt about the existence of subjects of consciousness and of physical action, or about the existence of their agency. We are subjects of consciousness and of physical action ourselves, and we act physically, with our brains, with and for our organisms. There is only a question about the initial, or first, agency of a subject of consciousness and of physical action: How does that agency come about?

Counter-question: Must every instance of causality have a specified way in which it works? The answer is: Not every instance of causality can have a specified way in which it works. The situation is as follows: If a causal nexus is mediate, then how it works can, in principle, to some extent be elucidated. Some understanding of how it works can be achieved by showing

6 Does 'without superfluity of the Domindar's causality' mean that the brain event would not have happened if it had not been caused by the Domindar? Not necessarily; for even if it was caused without superfluity of the Domindar's causality (without unnecessary causal overdetermination coming from the Domindar's side), the brain event might perhaps have happened anyway, perhaps without any sufficient cause at all: 'by chance.' (The latter possibility is, however, excluded if the principle of sufficient cause is true.)

7 Not to everybody but to some people, it seems mysterious when the Domindar caused the brain event. But this is not mysterious at all: the Domindar caused the brain event at the time it happened, not earlier, not later. In turn, not to everybody but to some people, it seems mysterious what the function of the Domindar's causing of the brain event is; but, again, this is not mysterious at all: the Domindar's causing of the brain event is the initiation of the implementation of a choice between macroscopic physical possibilities, a choice regarding which of these possibilities will be actual. [Details can be found in (Meixner 2014).] 
that the nexus consists of several steps of cause and effect, where each cause and each effect is characterized in some detail; in other words, some functional understanding of a mediate causal nexus can be achieved by showing that the nexus is a causal chain, with such and such - specifically characterized - members. The finer the division and specification, the better is the explanatory result with respect to how the causal nexus works. However, due to human cognitive limitations, this procedure of discovery must inevitably come to an end. We must stop the procedure after a finite number of rounds (perhaps because we cannot see any further, perhaps because we simply have to go on with our lives), and thus the discovery and characterization of intermediate causes and effects terminates inevitably with causal nexuses where how they work is not - at least not yet - understood: the nexuses between items $\mathrm{X}_{\mathrm{i}}$ and $\mathrm{X}_{\mathrm{i}+1}$ in the causal chain that has so far-so far as one has come - been disclosed in scrutiny. If, however, a causal nexus is immediate - if it is without intermediate, mediating causes and effectsthen a division of the causal nexus into causal steps is impossible; here, one cannot discover any (proper) causal chains ${ }^{8}$ and no description of the given cause and effect, be it ever so detailed, will produce an understanding of how their causal connection works (that is, an understanding in addition to the, so to speak, trivial understanding which is already provided by the very definition of their relationship as one of cause and effect ${ }^{9}$ ). Here, one is confronted from the start with a causal nexus which cannot be functionally understood, not even to some extent. Now, the causality in which a nonphysical subject of consciousness and of physical action causes brain events which have no sufficient physical cause - this causality is a purely

8 Proper causal chains have $\mathrm{N}$ members, where $\mathrm{N} \geq 3: \mathrm{X}_{1} \rightarrow \mathrm{X}_{2} \rightarrow \mathrm{X}_{3} ; \mathrm{X}_{1} \rightarrow$ $\mathrm{X}_{2} \rightarrow \mathrm{X}_{3} \rightarrow \mathrm{X}_{4} ; \cdots$

9 Certain powers, or, alternatively, 'covering laws,' or, more esoterically, certain comparative similarities between possible worlds, may sometimes be sufficient for constituting a connection of cause and effect, but, by themselves, they do not provide any information about how it works: they may constitute the connection, but do not begin to make it functionally transparent. A thoroughly agent-causal conception of the causal connection can be found in (Meixner 2017); but of course this conception, too, arrives at its limits when the question is how the causal connection works. 
immediate one. It is, as a purely immediate one, also a purely first-causal one. ${ }^{10}$ This much, at least, can be perfectly understood about purely immediate causality. But there is nothing explanatory to be said about how it works, how it functions, what its mechanism is. It is an ontically effective choice. That's all.

\section{References}

Barrett, Jeffrey A. 2014. "Quantum Mechanics and Dualism." In Quantum Physics Meets the Philosophy of Mind. New Essays on the Mind-Body Relation in Quantum-Theoretical Perspective, edited by Antonella Corradini and Uwe Meixner, 65-81. Berlin: De Gruyter. https://doi.org/10.1515/9783110351064.65 Craig, William Lane, and James D. Sinclair. 2012. "The Kalam Cosmological Argument." In The Blackwell Companion to Natural Theology, edited by William Lane Craig and James Porter Moreland, 101-201. Malden, MA, and Oxford: Wiley-Blackwell. https://doi.org/10.1002/9781444308334.ch3

DeWitt, Bryce S.M., and R. Neill Graham, eds. 1973. The Many-Worlds Interpretation of Quantum Mechanics. Princeton: Princeton University Press.

Eccles, John C., and Karl R. Popper. 1977. The Self and Its Brain. Berlin: Springer. https://doi.org/10.1007/978-3-642-61891-8

10 Purely immediate and purely first-causal causation are logically equivalent: Let ' $\mathrm{C} a b$ ' stand for ' $a$ causes $b$,' ' $a$ is a sufficient cause of $b$.' $y$ is purely first-causally caused, for example by $x$ : $\mathrm{C} x y \wedge \forall z(\mathrm{C} z y \supset \neg \exists z \mathrm{C} z z)$; y is purely immediately caused, for example by $x$ : $\mathrm{C} x y \wedge \forall z\left(\mathrm{C} z y \supset \neg \exists z\left(\mathrm{C} z z^{\prime} \wedge \mathrm{C} z y\right)\right)$. Given these two definitions, it is true for all $x$ and $y$ : if $y$ is purely first-causally caused, for example by $x$, then $y$ is purely immediately caused, for example by $x$. [Assume: $\mathrm{C} x y \wedge \forall z(\mathrm{C} z y \supset \neg \exists z \mathrm{C} z z)$, and assume for reductio: $\neg(\mathrm{C} x y \wedge \forall z(\mathrm{C} z y \supset \neg \exists z(\mathrm{C} z z \wedge \mathrm{C} z y)))$; hence $\exists z(\mathrm{C} z y \wedge$ $\exists z(\mathrm{C} z z \wedge \mathrm{C} z y))$; hence $\exists z^{\prime}\left(\mathrm{C} z y \wedge \exists z \mathrm{C} z z^{\prime}\right)$-contradicting the first assumption.] It is an obvious corollary that it is true for all $y$ : if $y$ is purely first-causally caused $[\exists x \mathrm{C} x y \wedge \forall z(\mathrm{C} z y \supset \neg \exists z \mathrm{C} z z)]$, then $y$ is purely immediately caused $[\exists x \mathrm{C} x y \wedge \forall z(\mathrm{C} z y$ $\supset \neg \exists z(\mathrm{C} z z \wedge \mathrm{C} z y))]$. And also the converse of this is true for all $y$ : if $y$ is purely immediately caused, then $y$ is purely first-causally caused. [Assume: $\exists x \mathrm{C} x y \wedge \forall z(\mathrm{C} z y$ $\left.\supset \neg \exists z\left(\mathrm{C} z z^{\prime} \wedge \mathrm{C} z^{\prime} y\right)\right)$, and assume for reductio: $\mathrm{C} z y \wedge \exists z \mathrm{C} z z$; hence $\mathrm{C} z y \wedge \exists z\left(\mathrm{C} z^{\prime} z\right.$ $\wedge \mathrm{C} z y)$, and from this, by the transitivity of causation: $\mathrm{C} z y \wedge \exists z(\mathrm{C} z z \wedge \mathrm{C} z y)$, hence $\exists z^{\prime}\left(\mathrm{C} z y \wedge \mathrm{C} z^{\prime} z \wedge \mathrm{C} z y\right)$, hence $\exists z^{\prime}\left(\mathrm{C} z y \wedge \exists z^{\prime}\left(\mathrm{C} z z^{\prime \prime} \wedge \mathrm{C} z^{\prime \prime} y\right)\right)$-contradicting the first assumption.] 
Kim, Jaegwon. 1993. Supervenience and Mind. Selected Philosophical Essays. Cambridge: Cambridge University Press. https://doi.org/10.1017/CBO9780511625220

Meixner, Uwe. 2006. "Consciousness and Freedom." In Analytic Philosophy without Naturalism, edited by Antonella Corradini, Sergio Galvan, and E. Jonathan Lowe, 183-96. London: Routledge.

Meixner, Uwe. 2008. "New Perspectives for a Dualistic Conception of Mental Causation." Journal of Consciousness Studies 15 (1): 17-38.

Meixner, Uwe. 2014. "Of Quantum Physics and DOMINDARs." In Quantum Physics Meets the Philosophy of Mind. New Essays on the Mind-Body Relation in Quantum-Theoretical Perspective, edited by Antonella Corradini and Uwe Meixner, 17-34. Berlin: De Gruyter. https://doi.org/10.1515/9783110351064.17 Meixner, Uwe. 2017. "Agent-Causation-Neither Upward Nor Downward." In Philosophical and Scientific Perspectives on Downward Causation, edited by Michele Paolini Paoletti and Francesco Orilia, 278-95. New York and London: Routledge (Taylor \& Francis). 